
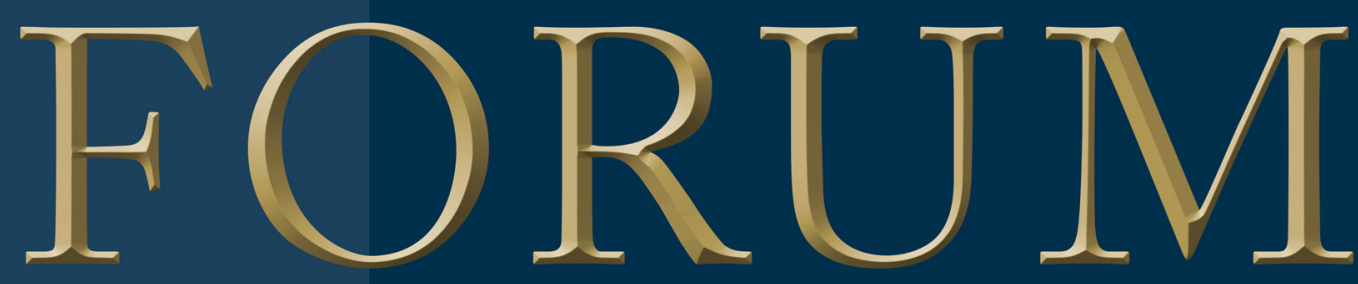

VOLUME 28 I NUMBER 1 JANUARY/FEBRUARY 2018

HAIR TRANSPLANT FORUM INTERNATIONAL

IN THIS ISSUE

The Successful Treatment of Alopecia Areata with Platelet Rich Plasma in a Case of Twins with Genetic Risk for Autoimmune Disorders

Advances in Robotic FUE

Spotlight on the ISHRS Ethics Committee

\title{
Redefining the "E" in FUE: Excision = Incision + Extraction
}

Ricardo Mejia, MD I Jupiter, Florida, USA I www.skinandhairdoc@aol.com

Extraction in the purest form can be defined as "the action of taking out something, especially using effort or force." In 2013, Dr. Parsa Mohebi and the FUE Research Committee published a report in the Forum (Vol. 23, No. 5, pp. 165-168) entitled, "Standardization of the terminology used in FUE: part I." In it, they noted that the concept of FUE was first published in the tabloid newspaper "The Sun Herald" in Australia on October 15, 1995, in an advertisement for Dr. Woods \& Dr. Campbell's top-up microsurgical technique where the donor extraction was done one follicular unit at a time. The advertisement described the concept of FUE as "Hair Follicle Single Unit Extraction." In 2002, Drs. Bill Rassman and Bob Bernstein published "Follicular Unit Extraction: Minimally Invasive Surgery for Hair Transplantation" (Dermatol Surg. 2002; 28(8): 720-727). They described the term FUE as "the removal of individual clusters of follicles from the donor area using a sharp dissecting punch or trephine." Drs. Rassman and Bernstein described the way $1 \mathrm{~mm}$-diameter punch incisions were made to separate the hair follicles and remove them.

In those early years, the key question for surgeons was: How do we remove the follicles? Hence, the word "extraction" was appropriately used. This term also provided a significant marketing advantage as

\section{Determining Safe Excision Limits in FUE: Factors That Affect, and a Simple Way to Maintain, Aesthetic Donor Density}

\author{
Sharon A. Keene, MD, FISHRS I Tucson, Arizona, USA I drkeene@hairrestore.com; \\ William R. Rassman, MD I Los Angeles, California, USA; James A. Harris, MD, FISHRS I \\ Denver, Colorado, USA
}

\section{Registration now open!}

\section{See page 33 .}

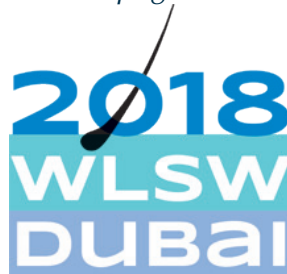

MARCH 8-10 DUBAI HEALTHCARE CITY

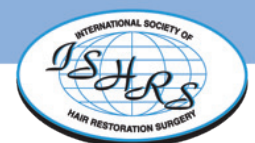

\section{Overharvesting and difficulties measuring} variables affecting donor coverage

The explosive worldwide popularity of the Follicular Unit Extraction (FUE) method for donor harvesting (recently re-defined to reflect the more appropriate surgical description, Follicular Unit Excision) has contributed to an increase in patients affected by donor area overharvesting. This has resulted in serious cosmetic defects ranging from minor degrees of visibly moth-eaten donor areas to almost complete donor alopecia. A separate, but related, problem occurs when areas of focal donor necrosis are created by overly aggressive FUE. Figure 1 illustrates examples of various donor defects that have been seen post-FUE. Safe excision guidelines to educate doctors to avoid these complications do not currently exist. In an effort to guide medical practitioners toward safe limits of FUE, the Hair Diameter Index ${ }^{1,2}$ and the Hair Coverage Value ${ }^{3}$ have been proposed to aid in predicting FUE harvest limits based on hair shaft diameters and hair count/square centimeter.
FIGURE 1. Donor areas in A and B illustrate focal scarring and alopecia following FUE; C illustrates a "mottled" donor area appearance.

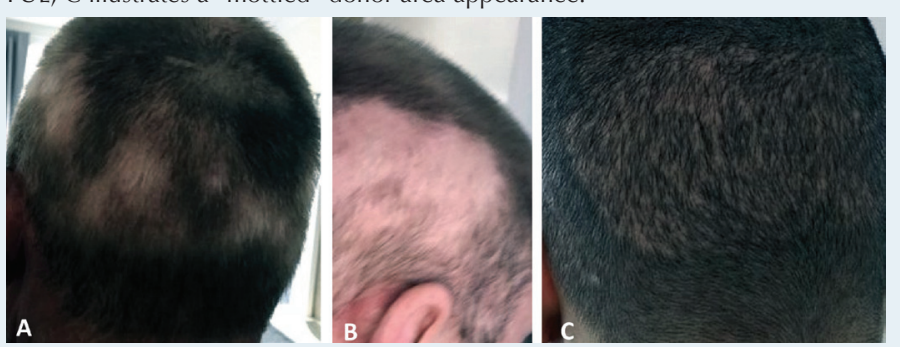


it did not imply surgery and was advertised in 1995 as it is today as "no scalpel, no stitches, no scar." However, as Dr. Mohebi and the Research Committee concluded, "In strict terminology, the term 'follicular unit extraction' is inappropriate and misleading because it is a histological term rather than an accurate anatomical surgical term."

So why do doctors continue to use the word extraction? The answer is simple. It's routine and accepted as the standard. It is very clear with simple mathematics what FUE surgeons do. We perform surgery to excise full-thickness skin grafts containing hair follicles. It all adds up, $1 / 2+1 / 2=1$, or: Incision + Extraction $=$ Excision .

Hence, a more appropriate and accurate term is Follicular Unit Excision. (The good news is that we can continue to refer to this procedure as "FUE," and it will always remain.)

Over the past 20+ years, there has been less focus on true extraction techniques and more focus on the incision aspect of the equation to minimize damage and transection rates and to obtain a better-quality graft. We have seen an explosion in the variety of "incision techniques" using handmade punches from 18- and 19-gauge needles, and sharp, serrated, non-serrated, dull, hybrid, Upunch, Trumpet punches, and more. A variety of automated devices also has evolved to assist with the speed of incisions, such as the S.A.F.E. System $^{\text {TM }}$, ARTAS $^{\circledR}$, NeoGraft ${ }^{\circledR}$, SmartGraft $^{\circledR}$, Vortex, PCID, WAW system, Atera, 3 Step FUE, RotoCore, Mamba, and other international devices. These devices, as well as many manual punch handles, have the ability to limit the depth of incisions.

As we continue to evolve and develop better-quality incision techniques, why do we continue to use inappropriate or misleading language? Simply put, it's a bad habit. The ATOE (Cole Instruments) —or Aide to Extraction-is one instrument that is appropriately named. To be precise and accurate in our communication, we should use the term "extraction" only when we are using techniques to physically manipulate and handle the graft to remove it from the body AFTER the incision is made. This can be done by suction, ATOE, the one-handed or two-handed technique, wiping grafts out using gauze, or other techniques that safely avoid damage to the graft. I see conferences and workshops advertising "extraction techniques" when all they are discussing is the way to properly cut the skin with the above incision techniques.

We are in the habit of using this term-extraction-and it will not be easily forgotten or changed. However, to use language in a precise, technically accurate way, we are advocating the change to Follicular Unit Excision. Excision embodies the true aspect of what we do as surgeons in both the academic and clinical aspect as it focuses on the two aspects of the equation: incision and extraction.

In addition, we have a responsibility for truth in advertising. Over the past 15 years, the term extraction has been minimized by many across the world to imply a non-surgical procedure that only involves "extracting" hairs as if they were being plucked out of the scalp without surgery. We continue to see advertisements that promise "no scar" or that use phrases such as "harvesting grafts," which minimize the procedure as if we are non-surgically gathering crops from a field. Given the worldwide expansion of this technique by non-medical and unlicensed personnel, the term extraction often is used to falsely mislead individuals so the procedure can be performed by non-medical personnel and to justify these actions to the public and legislators.

This is why I have proposed that hair transplant surgeons adopt Follicular Unit Excision as the new medical term. In a recent personal communication regarding the name change, both Drs. Rassman and Bernstein agree. Dr. Bernstein noted, "Times have changed and it will give more clarity to the term FUE and hopefully it will be more respected for the surgical procedure that it is." Many international FUE surgeons with whom I have discussed this also agree.

So how should we define FUE? We should define it to reflect the accuracy of the surgical implications:

Follicular Unit Excision is the surgical technique that refers to circumferential incision of the skin around the follicular unit bundle or group of hair follicles for the purpose of extracting a full-thickness skin graft containing hair follicle(s), intradermal fat, dermis, and epidermis.

The ISHRS Board of Governors has reviewed this new terminology and agreed that the above definition more accurately reflects the true nature of the procedure. It also prevents any type of misleading or fraudulent information that may be conveyed to the public. We have heard from leading physicians and textbook authors across the world that this updated terminology "makes sense," and that they are already making plans to incorporate the new culture and terminology into future textbooks. The ISHRS is also on board with making this part of our communication dialogue. Consequently, we are suggesting that the membership adopt this new terminology. Follicular unit incision and extraction techniques will never go away, but at least we can be more academically and clinically precise with our language and communication. I hope that each of you will join us in this transition as we bring in the New Year with Follicular Unit Excision for 2018 and beyond.

On page 6, please see what your colleagues are saying about this change from Extraction to Excision. 
Robert M. Bernstein and William R. Rassman began a chain of responses to this change of nomenclature:

This article on FUE name change adds significant clarity to the nomenclature of hair transplantation. Renaming Follicular Unit Extraction to Follicular Unit Excision acknowledges two distinct steps-incision and extraction-that will make communicating with our patients easier and more concise. It will also allow clinicians and researchers to think more clearly about the two steps of FUE, both separately and together, when addressing such issues as transection, suction injury, punch design, automation, and robotics. Although Shakespeare aptly pointed out that times a name can be quite irrelevant: "What's in a name? That which we call a rose by any other name would smell as sweet" [Romeo and Juliet, II, ii, 1-2], in this case the important change in wording should have lasting significance.

\section{Our current president:}

Sungjoo (Tommy) Hwang, MD, PhD, FISHRS: I think it is a great idea. FU Excision is a more scientific and medical term.

\section{Our past presidents:}

Jerry E. Cooley, MD, FISHRS: I think it's excellent

Paul C. Cotterill, MD: I definitely agree with the name change to Follicular Unit Excision. This important step will help to control the ambiguity that has been perpetuated and exploited inappropriately by some physicians and companies in our field. This new terminology-FUE: excision = incision + extraction-more accurately reflects the technique.

Edwin S. Epstein, MD: Well written and timely.

Bessam K. Farjo, MBChB: Congratulations on the excellent document you put together, and certainly the term "excision" is far more logical and correctly describes the process. It would have been almost impossible to change the acronym FUE, and so it is great that the suggested new terminology slots in perfectly!

Vincenzo Gambino, MD, FISHRS: Your draft is an excellent piece of scientific writing and truly clarifies a very important distinction that FUE is a surgical procedure.

Marcelo Gandelman, MD: Definitely Follicular Unit Excision! The repeating pattern in relation to the term FUE is in fact damaging our communication with patients. Your idea is innovative with a practical solution surely necessary for our colleagues both in an academic or professional environment. With this article, you are bonding your experience with innovation and have found the solution to the problem. As Dr. Bernstein would say: "Why didn't I think of that?"

Robert S. Haber, MD: While it is almost impossible to change a term once it has entered the public lexicon, it is still a sensible plan; I applaud the idea. By the way, I've been using the term FU Excision in my verbal discussions with patients since the concept was presented, and it was very easy to make the switch.

Sheldon S. Kabaker, MD: "Excision" seems to be a more accurate term than "extraction." All this is appropriate academically, and I support this subtle but more proper definition.

Sharon A. Keene, MD, FISHRS: I like the latest version, and agree it encompasses the important aspects of the technique-including the fact that the extraction does not preclude excision - so when people read this it seems quite clear incising and excising of tissue is occurring... Agree with the need for a definition that describes the important surgical aspects of the technique and is sufficiently broad to cover many different devicesand indicates that more than one hair follicle is often being removed.

Robert T. Leonard, Jr., DO: I wholeheartedly support the Board's decision to change the definition of the " $E$ " in FUE to Excision from Extraction. Hindsight is 20:20, isn't it? If this had been the initial definition from many years ago, our field would not be in the mess we find ourselves in today with unethical, inappropriate, and misleading advertising of this surgical harvesting technique coupled with the fact that non-surgeons are still excising tissue, i.e., performing surgery!

Jennifer H. Martinick, MBBS: The change to "excision" makes perfect sense as it encapsulates the total procedure; incise (a surgical procedure) plus extraction. Well done improving the nomenclature.

Mario Marzola, MBBS: I also agree that the name change better reflects the technique of FUE. It will be difficult to change an established name, but if we all band together, it will gain momentum. We are starting today!
James E. Vogel, MD: Of course this new terminology makes 100\% logical sense. Certainly I support it!!

Kuniyoshi Yagyu, MD, FISHRS: I agree with the idea of FU Excision. It is an accurate term of the procedure.

\section{Other comments}

Konstantinos K. Anastassakis, MD, PhD: Good idea.

Marco N. Barusco, MD, FISHRS: I think that the nomenclature change is very appropriate and scientifically correct.

Michael L. Beehner, MD, FISHRS: I welcome this change in terminology, since for too long some of the proponents of Follicular Unit Extraction have tried to portray to the public the idea that the procedure is done without any surgery or cutting of tissue. I also agree the change helps label the procedure for what it is, namely, surgery, and that non-physicians should not be performing this.

Kanokwan Chantauppalee, MD: I agree about the new terminology.

Ekrem Civas, MD, FISHRS: I completely agree with this change to excision. Extraction only describes the act of pulling out something, as if a punch incision was not made beforehand. The use of the word extraction simplifies the perception of the procedure, that it can easily be done by anyone and not a hair surgeon; extraction is not a scientifically sufficient academic term.

Ivan S. Cohen, MD, FISHRS: Redefining the " $E$ " in FUE to mean Excision rather than Extraction is a brilliant idea. It defines what we do more accurately, which will help the public understand that this is in fact a surgical procedure.

James A. Harris, MD, FISHRS: FUE as commonly performed is in fact an excision. Excision covers it all...whether rotary, oscillation, sharp or blunt, ultrasound or laser...partial or full depth.

Chiara Insalaco, MD, PhD: The new term synthesizes perfectly what technically happens during the FUE hair restoration. I hope it can be a start towards a big change in this, unfortunately, wild field.

Paul J. McAndrews, MD, FISHRS: For the public to be deceived that FUE is an extraction (not excision) with the implication that it is not really a surgery and only gives you "white dots" is wrong. I absolutely agree. The only difference between the punch excision done in the 1960s and FUE of today is the size of the punch. The total surface area of scar tissue created per follicular unit removed is actually greater for a $1 \mathrm{~mm}$ FUE punch versus a $4 \mathrm{~mm}$ punch. Unfortunately, that is not great for marketing.

Osman T. Oguzoglu, MD: I think it's very good idea. I will change all FUE extraction to FUE excision in my website, because patients will think it's a more complicated process and should be done by a doctor.

David Perez-Meza, MD, FISHRS: I agree and I support the proposal about FU Excision. I and others discussed the terminology "excision" with Dr. Crassas 18-19 years ago at the 1999-2000 Orlando Live Surgery Workshop.

Marcelo Pitchon, MD: I consider the change is pertinent and welcome. It is one of the elements necessary to make patients and the general public correctly informed that FUE is real surgery. And that it is not excisionfree, nor sequelae-free, nor riskless, nor scarless.

Nicole E. Rogers, MD, FISHRS: Wow! What a great concept! I think this is very helpful and will definitely clarify the concept that FUE is still surgery, not just "extraction" (sounds simple, non-surgical?) of hair follicles.

Antonio Ruston, MD: My opinion is that you are absolutely rightexcision is the correct terminology and defines better and more accurately the procedure (incision + extraction), and besides that, I agree that would prevent misleading or fraudulent information.

Arthur Tycosinski, MD, FISHRS: The name change is a master idea: Bingo! I totally support it.

Robin Unger, MD: I agree wholeheartedly. It is FU excision when the skin is cut. Extraction is removing them after the surgical aspect has been completed. And it does also clarify the need for the procedure to be done by trained medical personnel.

Michael W. Vories, MD: I agree that excision is a more precise term. If this at least has the possibility of defining the procedure as a surgical procedure, then I am all for it.

Sara M. Wasserbauer, MD, FISHRS: I am on board.

Ken L. Williams, DO, FISHRS: The nomenclature suggested by you I think is very good. It makes sense. As long as Bernstein, Rassman, and Rose are on board, I don't think there should be any problem in adopting this new language in our future FUE textbook. I like it.

Jerry Wong, MD: I agree that it is better defined as follicular unit excision. 